\title{
Intra-Operative Damage to the Pelvic Diaphragm Musculature and Difficulty in Exposure of the Urethra Are Risk Factors of Postoperative Urinary Incontinence after Laparoscopic Radical Prostatectomy: Review of Surgical Video
}

\author{
Yukihiro Umemoto Keiichi Tozawa Yoshiyuki Kojim Takahito Yasui \\ Noriyasu Kawai Shoichi Sasaki Yutaro Hayashi Kenjiro Kohri \\ Department of Nephro-urology, Nagoya City University Graduate School of Medical Sciences, Nagoya, Japan
}

\section{Key Words}

Laparoscopic radical prostatectomy • Urinary incontinence • Prostate cancer $\cdot$ Pelvic diaphragm musculature $\bullet$ Urethra

\begin{abstract}
Purpose: The aim of this study was to clarify risk factors related to postoperative urinary incontinence after laparoscopic radical prostatectomy (LRP). Patients and Methods: Outcomes of 214 consecutive patients who underwent LRP at our institute between April 2001 and January 2009 were reviewed. Patients were divided into 2 groups, continent patients (group A: $n=172$ ), who used one or fewer urinary pads per day 6 months after LRP, and incontinent patients (group $B: n=42$ ), who used two or more pads per day 6 months after LRP. Patient age, prostate specific antigen before LRP, blood loss, duration of indwelling urethral catheter, and the positive margin rate between the 2 groups were compared. In addition, surgical videos were reviewed with attention paid to surgical procedures. We examined the correlation between the occurrence of urinary incontinence and the rate of patients with intra-operative damage to the pelvic diaphragm musculature and difficulty in exposure of the urethra during LRP. Results: There were no significant dif-
\end{abstract}

\section{KARGER}

Fax +4161306 1234

E-Mail karger@karger.ch

www.karger.com
(C) 2011 S. Karger AG, Basel

1015-9770/11/0054-0185\$26.00/0

Accessible online at:

www.karger.com/cur ferences in patient age, prostate specific antigen before LRP, blood loss, duration of indwelling urethral catheter, positive margin rate between groups A and B. However, significant differences were found in the rate of patients with damage to the pelvic diaphragm musculature (16.3 and $73.8 \%$, respectively) and difficulty in exposure of the urethra (20.9 and $83.3 \%$, respectively) during LRP. Conclusions: Intra-operative damage to the pelvic diaphragm musculature and difficulty in exposure of the urethra during LRP are risk factors of urinary incontinence after LRP.

Copyright $\odot 2011$ S. Karger AG, Basel

\section{Introduction}

Laparoscopic radical prostatectomy (LRP), regardless of whether performed with or without a robotic assistant, is rapidly becoming an acceptable procedure as a minimally invasive alternative to open retro-pubic radical prostatectomy (RRP) for the treatment of localized prostate cancer, and has proved to be a safe and reliable sur- 

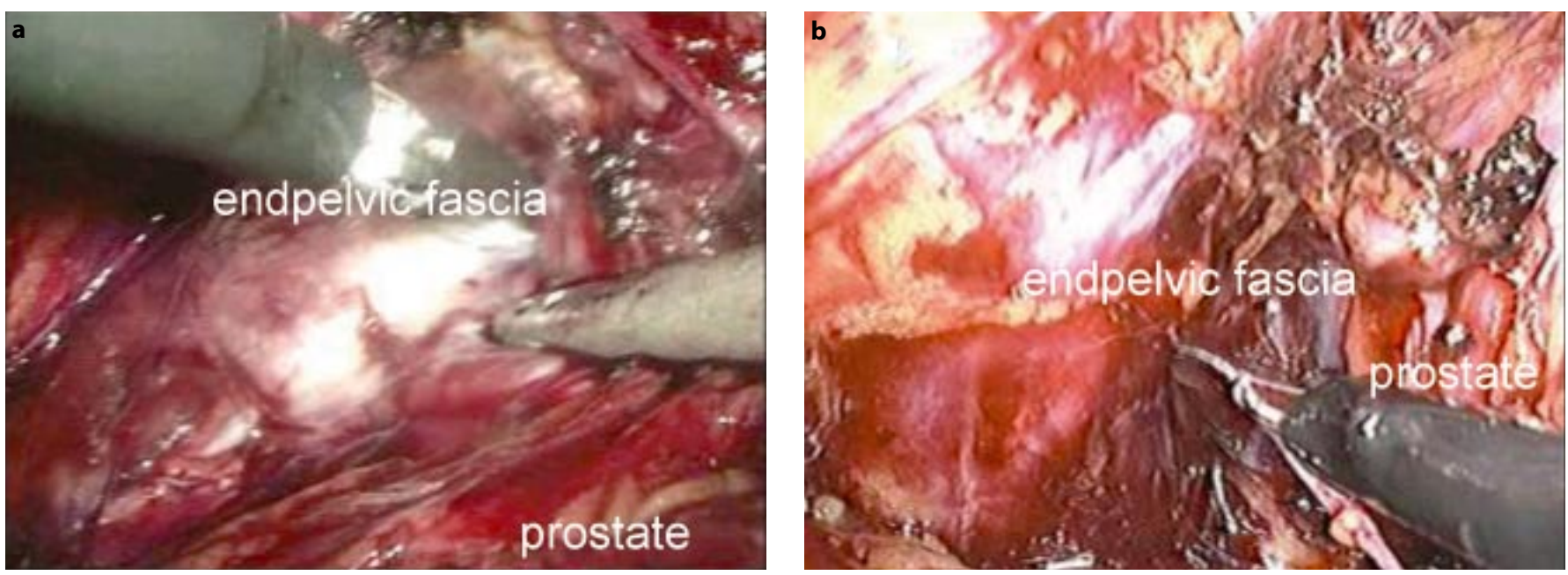

Fig. 1. Intra-operative findings in the left endopelvic fascia during LRP. a Clear exposure of the pelvic diaphragm musculature; $\mathbf{b}$ Difficulty in exposing the endopelvic fascia and, consequently, damage to the pelvic diaphragm musculature, such as levator ani musculature by coagulation injury.

gical procedure. Postoperative urinary incontinence is a common complication following radical prostatectomy. LRP results in minimal bleeding and magnification of the operative field, which theoretically could allow precise dissection of the prostatic apex with limited trauma to the peri-urethral striated sphincter and genitourinary diaphragm. Therefore, LRP was expected to reduce the occurrence of postoperative urinary incontinence. However, whether postoperative continence is improved with LRP compared with an open surgical approach remains controversial [1-4]. Although many investigators have proved the importance of preserving the pelvic diaphragm musculature and external urethral sphincter and its related structures to avoid postoperative urinary continence, the patient characteristics and anatomic findings in the prostate and its surrounding structures when performing LRP vary among patients. We considered that it was important to examine not only patient characteristics but also anatomic findings by reviewing the surgical video in order to clarify the risk factors of urinary incontinence after LRP.

In this study, we first examined the correlation between patient characteristics such as age, prostate specific antigen (PSA) before LRP, intra- and post-operative findings, such as blood loss, duration of the indwelling urethral catheter and the positive margin and postoperative urinary incontinence to clarify possible risk factors of postoperative urinary incontinence after LRP. Then surgical videos were reviewed with attention paid to the surgical procedure in each patient, and we examined the correlation between postoperative urinary incontinence and intra-operative damage to the pelvic diaphragm musculature or difficulty in exposure of the urethra during LRP.

\section{Patients and Methods}

Outcomes of 214 consecutive patients who underwent LRP for localized prostate cancer (T2-T3NOM0) at our institute between April 2001 and January 2009 were reviewed. Indications for LRP were patients with clinical stage T1c-T2c with a life expectancy of more than 10 years. The LRP procedure was based on the Montsouris technique with some modifications [5, 6].

Six months after LRP, the interviewing surgeon asked the patients about the number of pads used. The patients were divided into 2 groups, continent patients (Group A: $n=172$ ), who used one or fewer urinary pads per day 6 months after LRP, and incontinent patients (Group B: $n=42$ ), who used two or more pads per day 6 months after LRP.

First, we compared the patient age, PSA before LRP, blood loss, duration of indwelling urethral catheter, and the positive margin rate between the 2 groups to identify the risk factors of urinary incontinence after LRP. In addition, surgical videos were reviewed with attention paid to surgical procedures. We focused on surgical findings in the exposure of endopelvic fascia and the urethra during LRP. 

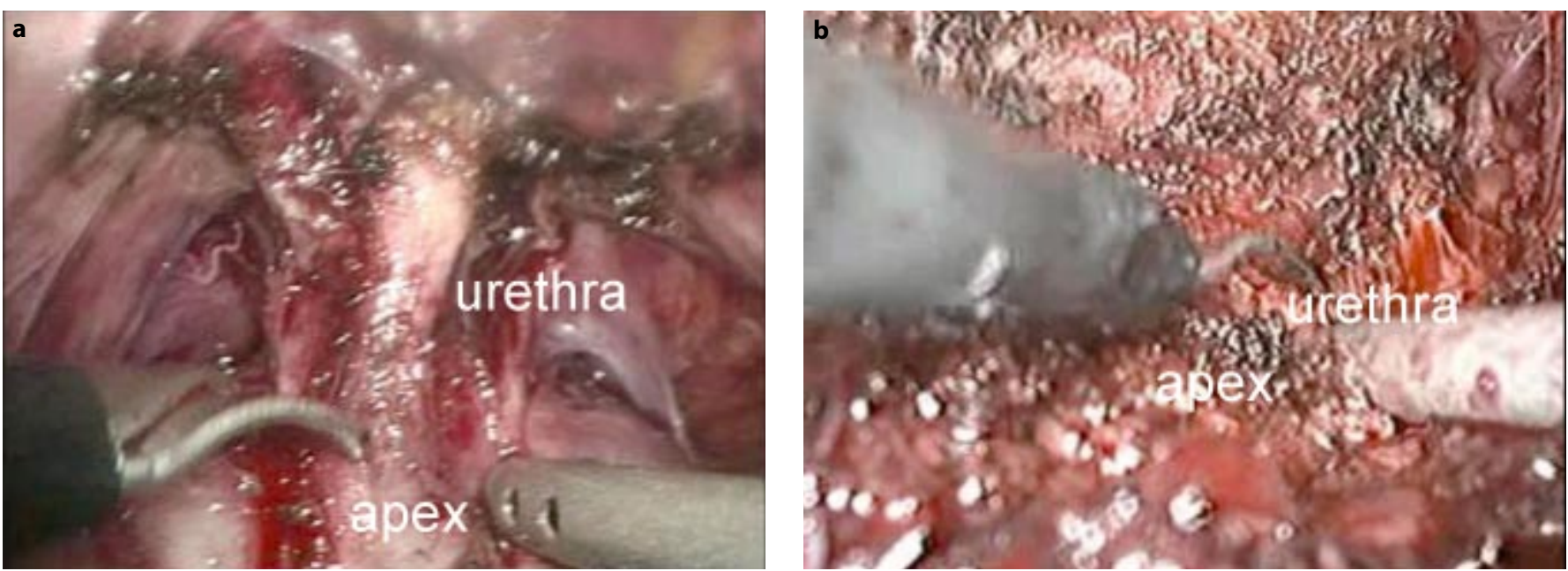

Fig. 2. Intra-operative findings in prostatourethral junction during LRP. a Good visualization of the prostatourethral junction; b Bleeding from the dorsal vein complex and difficulty in exposing the urethra.

We dissected the fibro-adipose tissue covering the prostate to expose the endopelvic fascia, made an incision in the endopelvic fascia and extended the incision toward the puboprostatic ligaments. At this stage, small arterial and venous branches from the pudendal vessels that perforate the pelvic musculature to supply the prostate were often encountered. We usually succeeded in detaching the prostate and bladder from the endopelvic fascia by incising the endopelvic fascia and clearly exposing the pelvic floor muscle (fig. 1a). However, injury to small arterial and venous branches from the pubodendal vessels resulted in difficulty in exposing the endopelvic fascia, leading to the use of electrocautery to control bleeding from these vessels and, consequently, damage to the pelvic diaphragm musculature, such as the levator ani musculature, by coagulation injury (fig. 1b). If we detected injury findings in the bundle of muscle fibers in the pelvic diaphragm by electrocautery coagulation on the surgical video, we judged it as damage to the pelvic diaphragm musculature.

After division of the dorsal vein complex, the prostatourethral junction was usually well visualized (fig. 2a). However, bleeding from the dorsal vein complex could result in difficulty in exposing the urethra (fig. 2b) and consequently, possible injury to the surrounding striated sphincter. If we did not find a long urethra, which was exposed from the distal to apex of the prostate to the pelvic floor, on the surgical video, we judged it as difficult to expose the urethra.

In this study, we examined the correlation between the occurrence of incontinence and the rate of patients with intra-operative damage to the pelvic diaphragm musculature or difficulty in exposing the urethra during LRP.

Student's t-test was used to test any significant differences in continuous variables between the 2 groups, and Fisher's exact test was used to analyze categorical data, including the continence rate for statistical significance. Statistical significance was defined as $\mathrm{p}<0.05$.

Risk Factors of Urinary Incontinence after LRP
Table 1. Patient characteristics

\begin{tabular}{lccc}
\hline & Group A & Group B & p \\
\hline Patients, $\mathrm{n}$ & 172 & 42 & \\
Age, years & $66.7 \pm 5.9$ & $67.7 \pm 6.1$ & n.s. \\
PSA before LRP, ng/ml & $11.7 \pm 12.2$ & $11.2 \pm 6.0$ & n.s. \\
Blood loss included urine, ml & $584 \pm 510$ & $602 \pm 528$ & n.s. \\
Indwelling urethral catheter, days & $8.0 \pm 3.7$ & $8.8 \pm 6.8$ & n.s. \\
Positive margin rate, \% & 31.4 & 38.1 & n.s. \\
\hline
\end{tabular}

n.s. = Not significant .

\section{Results}

First, we compared the patient age, PSA before LRP, blood loss, duration of the indwelling urethral catheter, and the positive margin rate between the 2 groups, and patients with or without postoperative urinary incontinence, to identify the risk factors of urinary incontinence after LRP. There were no significant differences in patient age, PSA before LRP, blood loss, duration of the indwelling urethral catheter, and the positive margin rate between Groups A and B (table 1).

Next, the correlation between postoperative urinary incontinence and intra-operative damage to the pelvic diaphragm musculature or difficulty in exposing the ure-

Curr Urol 2011;5:185-189 
thra during LRP by review of the surgical video were examined. The rate of patients with damage to the pelvic diaphragm musculature was significant lower in group A than group B $(16.3$ vs. $83.7 \%, p<0.05)$. Additionally, the rate of procedures (not patients) with difficulty in exposing the urethra was significantly lower in group A than group B (20.9 vs. $79.1 \%, \mathrm{p}<0.05)$.

\section{Discussion}

Prostate cancer accounts for approximately one third of cancer in men, and $86 \%$ of diagnosed prostate cancer occurred in the United States in 2004 [7]. In Japan, prostate cancer has increased, as in the USA, and the number of radical prostatectomy for patients with prostate cancer is increasing. Radical prostatectomy for localized prostate cancer is considered a safe and reliable surgical procedure, but urinary incontinence is a common complication following radical prostatectomy.

The frequency of urinary incontinence following open RRP can be in a range of 2-65\% [8]. Many comparative studies have confirmed the advantages of laparoscopic surgery in terms of blood loss, transfusion rates, postoperative pain scores, and the duration of both catheterization and hospitalization, with similar peri-operative complication rates to those of open prostatectomy. Potentially, however, better long-term quality of life may be achieved with LRP, and several studies have shown no significant difference in the incidence of urinary incontinence between RRP and LRP [5, 9]. On the other hand, Salomon et al. [10] reported no significant difference in continence at 1 year, but patients undergoing LRP had an earlier return to continence in the prospective period in comparison to patients with open and LRP. Whether postoperative continence is improved with LRP compared with the open surgical approach remains controversial [1-4]. In this study, patient age, PSA before LRP, blood loss, operation site, duration of the indwelling urethral catheter, and the positive margin rate were selected as possible risk factors of urinary incontinence between continent and incontinent patients after LRP. Generally, the preservation of urinary continence after radical prostatectomy is associated with patient age. However, patient age was not a significant risk factor for urinary incontinence in our study. In addition, other parameters, such as PSA before LRP, blood loss, operation site, duration of the indwelling urethral catheter, and the positive margin rate were not risk factors for urinary incontinence.
The etiology of urinary incontinence following radical prostatectomy is multi-factorial [11], although bladder neck dysfunction, detrusor instability, external sphincteric damage and damage to the pelvic diaphragm musculature (levator ani and deep transversus perinea) have been reported. The disruption of suspensory structures within the pelvis, and the external urethral sphincter and its related structures has been shown to be the main cause of postoperative urinary incontinence [12]. Therefore, we considered that preservation of the function of the pelvic diaphragm musculature and urethral sphincter as most important to maintain urinary continence after radical prostatectomy.

The advantage of LRP over RRP is the ability to review the surgical findings after operation from the same view as during the operation, and to clarify precisely the surgical problems after the operation. In this study, we reviewed the surgical videos of all patients retrospectively and focused on the surgical findings of the endopelvic fascia and urethra in order to examine the possible cause of urinary incontinence in LRP. The main points observed were whether we could preserve the pelvic diaphragm musculature and clearly expose the urethra. As a result, the rate of patients with damage to the pelvic diaphragm musculature and difficulty in clear exposure of the urethra during LRP in patients with urinary incontinence was significantly higher than in patients without urinary incontinence. Difficulty in clear exposure of the urethra during LRP could result in damage to the urethra and its surrounding musculature immediately distal to the apex of the prostate. These results suggest that it is imperative to preserve as much of the pelvic diaphragm musculature and urethra and its surrounding musculature as possible. However, a weak point of the present study is that it was non-blind and retrospective. Additionally, continence was not defined using a validated questionnaire. Although the study showed that intra-operative damage to the pelvic diaphragm musculature and difficulty in exposing the urethra are risk factors of postoperative urinary incontinence after LRP, further study will be needed to clarify the definitive risk factors of postoperative incontinence.

Developing technical modifications of radical prostatectomy that will lead to a decreased incidence of urinary incontinence has become a major priority for many urologists [13-16]. Many methods of preventing urinary incontinence have been reported, including nerve-sparing [13], puboprostatic ligament sparing [14, 15], bladder neck preservation $[14,16]$, and reconstruction of the posterior aspect of the rhabdosphincter [17-19]. Although 
these methods can possibly prevent urinary incontinence after radical prostatectomy, they may not be necessary in all cases. Surgical modification to avoid urinary incontinence as well as efforts not to injure the pelvic diaphragm musculature and urethra are needed to preserve quality of life in patients who have undergone LRP.

\section{Conclusion}

From the results of 214 cases, intra-operative damage to the pelvic diaphragm musculature and difficulty in exposing the urethra during LRP are risk factors for urinary incontinence after LRP. Since there are many methods of preventing urinary incontinence after LRP, they should be implemented when there is a possibility of urinary incontinence after LRP.

\section{References}

$\checkmark 1$ Ficazzola MA, Nitti VW: The etiology of post-radical prostatectomy incontinence and correlation of symptoms with urodynamic findings. J Urol 1998;160:1317-1320.

2 Chao R, Mayo ME: Incontinence after radical prostatectomy: detrusor or sphincter causes. J Urol 1995;154:16-18.

-3 Gomha MA, Boone TB: Voiding patterns in patients with post-prostatectomy incontinence: urodynamic and demographic analysis. J Urol 2003;169:1766-1769.

4 Matsukawa Y, Hattori R, Komatsu T, Funahashi Y, Sassa N, Gotoh M: De novo detrusor under-activity after laparoscopic radical prostatectomy. Int J Urol 2010;17:643-648.

$\checkmark 5$ Guillonneau B, Vallancien G: Laparoscopic radical prostatectomy: the Montsouris experience. J Urol 2000;163:418-422.

6 Tozawa K, Hashimoto Y, Yasui T, Itoh Y, Nagata D, Akita H, Kawai N, Hayashi Y, Kohri K: Evaluation of operative complications related to laparoscopic radical prostatectomy. Int J Urol 2008;15:222-225.

>7 Finkelstein J, Eckersberger E, Sadri H, Taneja SS, Lepor H, Djavan B: Open versus laparoscopic versus robot-assisted laparoscopic prostatectomy: the European and US experience. Rev Urol 2010;12:35-43.

$>8$ Carlson KV, Nitti VW: Prevention and management of incontinence following radical prostatectomy. Urol Clin North Am 2001;28: 595-612.
-9 Jacobsen NE, Moore KN, Estey E, Voaklander D: Open versus laparoscopic radical prostatectomy: a prospective comparison of postoperative urinary incontinence rates. J Urol 2007;177:615-619.

10 Salomon L, Levrel O, de la Taille A, Anastasiadis AG, Saint F, Zaki S, Vordos D, Cicco A, Olsson LE, Hoznek A, Chopin D, Abbou CC: Radical prostatectomy by the retropubic, perineal and laparoscopic approach: 12 years of experience in one center. Eur Urol 2002; 42:104-110.

11 Kim IY, Hwang EA, Mmeje C, Ercolani M, Lee DH: Impact of prosterior urethral plate repair on continence following robot-assisted laparoscopic radical prostatectomy. Yonsei Med J 2010;51:427-431.

12 Hammerer P, Huland H: Urodynamic evaluation of changes in urinary control after radical retropubic prostatectomy. J Urol 1997;157: 233-236.

13 Hollabaugh RS Jr, Dmochowski RR, Kneib TG, Steiner MS: Preservation of putative continence nerves during radical retropubic prostatectomy leads to more rapid return of urinary continence. Urology 1998;51:960 967.

14 Deliveliotis C, Protogerou V, Alargof E, Varkarakis J: Radical prostatectomy: bladder neck preservation and puboprostatic ligament sparing-effects on continence and positive margins. Urology 2002;60:855-858.
15 Poore RE, McCullough DL, Jarow JP: Puboprostatic ligament sparing improves urinary continence after radical retropubic prostatectomy. Urology 1998;51:67-72.

-16 Srougi M, Nesrallah LJ, Kauffmann JR, Nesrallah A, Leite KR: Urinary continence and pathological outcome after bladder neck preservation during radical retropubic prostatectomy: a randomized prospective trial. J Urol 2001;165:815-818.

$\checkmark 17$ Rocco F, Carmignani L, Acquati P, Gadda F, Dell'Orto P, Rocco B, Bozzini G, Gazzano G, Morabito A: Restoration of posterior aspect of rhabdosphincter shortens continence time after radical retropubic prostatectomy. $\mathrm{J}$ Urol 2006;175:2201-2206.

18 Rocco F, Carmignani L, Acquati P, Gadda F, Dell'Orto P, Rocco B, Casellato S, Gazzano G, Consonni D: Early continence recovery after open radical prostatectomy with restoration of the posterior aspect of the rhabdosphincter. Eur Urol 2007;52:376-383.

19 Rocco B, Gregori A, Stener S, Santoro L, Bozzola A, Galli S, Knez R, Scieri F, Scaburri A, Gaboardi F: Posterior reconstruction of the rhabdosphincter allows a rapid recovery of continence after transperitoneal videolaparoscopic radical prostatectomy. Eur Urol 2007;51:996-1003. 


\section{Erratum}

A spelling error occurred in the article: Umemoto et al: Intra-Operative Damage to the Pelvic Diaphragm Musculature and Difficulty in Exposure of the Urethra Are Risk Factors of Postoperative Urinary Incontinence after Laparoscopic Radica Prostatectomy: Review of Surgical Video. Curr Urol 2011;5:185-189. The third author Yoshiyuki Kojim should read Yoshiyuki Kojima. 